\title{
STRUCTURE OF 15 HECTARES PERMANENT PLOTS OF TERRA FIRME DENSE FOREST IN CENTRAL AMAZON ${ }^{1}$
}

Katia Emidio da Silva², Sebastião Venâncio Martins ${ }^{3 *}$, Carlos Antônio Alvares Soares Ribeiro ${ }^{3}$, Nerilson Terra Santos ${ }^{4}$ and Celso Paulo de Azevedo ${ }^{2}$

\footnotetext{
${ }^{1}$ Received on 24.10.2013 accepted for publication on 01.12.2015.

${ }^{2}$ Embrapa Amazônia Ocidental, Manaus, Amazonas - Brasil. E-mail: <katia.emidio@embrapa.br $>$ and <celso.azevedo@embrapa.br>. ${ }^{3}$ Universidade Federal de Viçosa - Departamento de Engenharia Florestal, Viçosa, MG - Brasil. E-mail: <venancio@ufv.br> and <cribeiro@ufv.br>.

${ }^{4}$ Universidade Federal de Viçosa, Centro de Ciências Exatas e Tecnológicas, Viçosa, MG - Brasil. E-mail: <nsantos@ufv.br>. *Corresponding author.
}

\begin{abstract}
Terra Firme dense forest occurs in $65 \%$ of the Amazonian region and is characterized by the high diversity of plant species and high occurrence of rare plant species. The objective of this work was to analyze the horizontal structure of 15 hectares of plots, randomly chosen from a set of 400 ha of permanent plots situated at the Experimental Site of Embrapa, Manaus, Amazonas State, Brazil. All individuals with $\mathrm{DBH} \geq 10 \mathrm{~cm}$ were identified at the level of species in 2005. The VI values (Values of importance) were separated in classes of range with one (01) standard deviation and the individuals distributed according to DBH classes of $10 \mathrm{~cm}$ in amplitude. In total, 8771 individuals were identified, distributed into 264 species and 53 families. A larger number of individuals is concentrated in a few number of species, such as Protium hebetatum D.C. Daly, Eschweilera coriacea (DC.) S.A. Mori and Licania oblongifolia Standl, which sum up $21 \%$ of the total number of individuals and $12 \%$ of the VI. There is a high occurrence of rare species (36\%). The families Sapotaceae, Lecythidaceae and Burseraceae together accounted for $39 \%$ of the total number of individuals. The diametric structure in an inverse-J shape shows that $80 \%$ of the individuals are concentrated in the DBH classes ranging from 10 to $30 \mathrm{~cm}$. The results highlight the high occurrence of rare species and those with low density, and with distribution restricted to some plots, particularly those belonging to the lowest classes of VI, which deserve special attention in actions for biodiversity conservation and forest management.
\end{abstract}

Keywords: Phytosociology; Tree species; Amazon.

\section{ESTRUTURA DO COMPONENTE ARBÓREO EM 15 HA DE PARCELAS PERMANENTES DE FLORESTA DENSA DE TERRA FIRME NA AMAZÔNIA CENTRAL}

\begin{abstract}
RESUMO - A floresta densa de terra firme ocorre em $65 \%$ da região Amazônica e é caracterizada por possuir alta diversidade de espécies vegetais e elevada ocorrência de espécies raras. Este trabalho analisou a estrutura horizontal de 15 ha de parcelas aleatoriamente selecionadas de um conjunto de 400 ha de parcelas permanentes, localizadas no Campo Experimental da Embrapa em Manaus, AM.Todos os indivíduos com DAP $\geq 10 \mathrm{~cm}$ foram identificados em nível de espécie em 2005. Os valores de VI (Valor de Importância) foram separados em classes de amplitude de um (01) desvio padrão e os individuos distribuídos segundo classes de DAP de amplitude de $10 \mathrm{~cm}$. Ao todo foram identificados 8771 indivíduos, distribuídos em 264 espécies e 53 famílias. Um maior número de indivíduos concentra-se em poucas espécies, tais como Protium hebetatum D.C.Daly, Eschweilera coriacea (DC.) S.A. Mori e Licania oblongifolia Standl. que somaram 21\% do número total de individuos e $12 \%$ do VI. Há elevada ocorrência de espécies raras (36\%). As familias Sapotaceae, Lecythidaceae
\end{abstract}




\begin{abstract}
e Burseraceae somaram 39\% do número total de indivíduos. A estrutura diamétrica em forma de J-invertido mostra que $80 \%$ dos individuos estão concentrados nas classes de 10-30 cm de DAP. Os resultados chamam a atenção para a elevada ocorrência de espécies raras e daquelas com baixa densidade, e com distribuição restrita a algumas parcelas, principalmente aquelas pertencentes às menores classes de VI, as quais devem merecer atenção especial nas ações de conservação da biodiversidade e manejo florestal.
\end{abstract}

Palavras-chave: Fitossociologia; Espécies arbóreas; Amazônia.

\section{INTRODUCTION}

Brazil, a country with approximately one-third of the remaining rain forests in the world, is one of the most important repositories of global biodiversity (PAS 2008). In the Amazon, many areas are formed by mosaic of habitats, with different sets of plant species occurring in adjacent areas on different substrates. It can be seen, in general, , that the distribution of tropical tree species can occur due to habitat preferences and/or variations in their evolutionary history (PITMAN et al., 2001; NOVAES FILHO et al., 2007).

The dynamics and structure of rain forests vary across the Amazon basin in an east-west gradient, following a pattern that matches variations in soil fertility (QUESADA et al., 2009). To describe these patterns of variation, using large data sets, such as those in the present study, is extremely important to enhance the understanding of the mechanisms that determine the distribution of species in nature (CORONADO et al., 2009).

About $65 \%$ of the Amazon region is covered by a forest type named terra firme dense forest. This plant typology is the most representative of the Amazon rainforest, mainly characterized by high richness and diversity of species with few individuals of each species and generally showing high dissimilarity floristic between adjacent plots (FERREIRA; PRANCE, 1998; LIMA FILHO et al., 2001; CONDÉ; TONINI, 2013).

Oliveira and Amaral (2004) reported the occurrence of 239 species in a study on one (01) hectare of terra firma dense forest in Manaus, evidencing the high diversity in this typology. Climatic and soil factors such as more humid climates and less seasonal and soils relatively fertile in nutrients, have been cited as possible responsible for a greater diversity of plants in the Western Amazon (GENTRY, 1988, QUESADA et al., 2009). Other authors such as Phillips et al. (1994) report the higher species richness to the natural dynamics of mortality of trees, where forests with high rates of mortality and recruitment would be more diversified.
Most studies in the Amazon uses, on average, one sample area of one (01) hectare for studies of floristic composition and structure. This study contributes to this subject, using a large data set. Thus, the objective of this study was to describe the horizontal structure of 15 hectares of terra firma dense forest in central Amazonia, Manaus, state of Amazonas, in order to contribute to enhance the knowledge on the organization of species in the community, therefore, helping in forest conservation actions and forest management in the Amazon.

\section{MATERIALAND METHODS}

\subsection{Study area}

The study area is located in a terra firme dense forest in Central Amazonia, in the Experimental Field of Embrapa Amazônia Ocidental, Manaus, in the state of Amazonas, Brazil, at the coordinates $2^{\circ} 32^{\prime} 12.75^{\prime \prime}$ $\mathrm{S} ; 60^{\circ} 0^{\prime} 14.62^{\prime \prime} \mathrm{W}$. The area comprises the project developed in the scope of Rede de Monitoramento da Dinâmica de Florestas da Amazônia BrasileiraREDEFLOR (Monitoring Net of Brazilian Amazon Forest Dinamics).

The weather in the area is "Am" type according to Köppen classification, with annual rainfall of 1,355 to $2,839 \mathrm{~mm}$. Average annual temperature is $27.6^{\circ} \mathrm{C}$, with air relative humidity ranging from 84 to $90 \%$ over the year. The most humid months are from December to May, and the driest ones are from July to October, when those months receive less than $100 \mathrm{~mm}$ of rain (RADAM, 1978).

The average altitude ranges from 60 to $160 \mathrm{~m}$ (REGIS, 1993). The predominant soils in the area are Yellow Latosol with texture ranging from more clayey on the plateaus to sandy in the lower parts. They are soils predominantly covered by vegetation of terra firme dense forest, with emergent canopy (IBGE, 1999), consisting of trees ranging from medium to large sizes, reaching up to $55 \mathrm{~m}$ of height (REGIS, 1993). 


\subsection{Data collection and analysis}

The plots were delimited at random in the Experimental Field, representing 15 plots of $100 \times 100$ $\mathrm{m}$. All individuals with diameter at breast height (DBH) e" $10 \mathrm{~cm}$ were labeled and botanically identified at the species level in 2005. The plant material was identified by means of comparisons with dried plants available in the herbarium of the Instituto Nacional de Pesquisas da Amazônia - INPA (National Institute of Amazonian Research) as well as by consulting the literature on native species of the Amazon (RIBEIRO et al., 1999). The botanical names were conferred at the Missouri Botanical Garden Web page (MOBOT, 2012), APGII system.

Calculation of the plant structural parameters, such as basal area (BA), absolute and relative dominance (ADo, RDo), abundance $(\mathrm{N})$, absolute and relative density (AD, RD) and absolute and relative frequency (AF, RF) for the composition of the Importance Value Index (IVI) were obtained according to Mueller-Dumbois and Ellenberg (1974), calculated on Mata Nativa 2 software (CIENTEC, 2006). The estimate of the ecological importance of families in the evaluated community was calculated by the Family Importance Index (FII), calculated by the sum of the diversity (number of species of the family/total number of species), relative density and dominance (MORI; BOOM, 1983) using the Microsoft Excel for Windows. Classes of IV (\%) were defined using for the upper limit of the first class, the average value minus 0.5 standard deviations (M-0.5* standard deviation) and thereafter, they were added with 1 standard deviation. The diameter classes were defined with an amplitude of $10 \mathrm{~cm}$.

\section{RESULTS}

Out of the fifteen sampled hectares, 8,771 individuals were identified, belonging to 264 species and 53 families. The number of trees and basal area per plot ranged from 497 to 688 and from 23.4 to $32.7 \mathrm{~m}^{2}$, respectively. The ten most important species in the study area, on the Importance Value (IV) basis, Figure 1, represent $24 \%$ of total IV, which were Protium hebetatum D.C. Daly, Eschweilera coriacea (DC.) S.A. Mori, Licania oblongifolia Standl., Pouteria minima T.D.Penn. and Ocotea cernua (Nees) Mez, the ones that presented the highest IV values. Only the first three species have $20.7 \%$ of the total number of identified individuals. The complete list of species, with their respective IV values is presented in the appendix.
The most important families, according to the Family Importance Value (FIV), in a decreasing order were Sapotaceae, Lecythidaceae, Burseraceae, FabaceaeMimosoideae and Chrysobalanaceae (Figure 2), in which

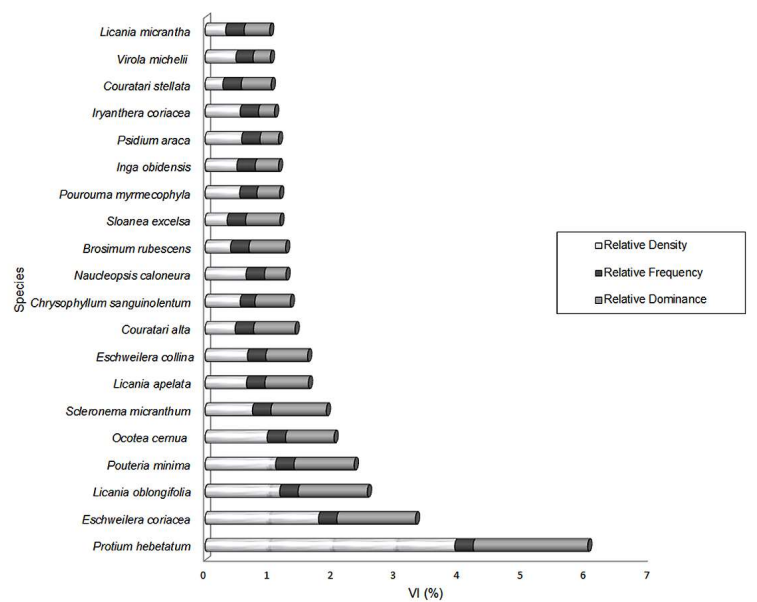

Figure 1 - Value index (VI\%) for the 20 species with the highest values at the Experimental site of Embrapa Amazônia Ocidental, Manaus, Amazonas State, Brazil.

Figura 1-Composição do Valor de Importância-VI(\%) para as 20 espécies com os maiores valores no Campo Experimental da Embrapa Amazônia Ocidental, Manaus, AM, Brasil.

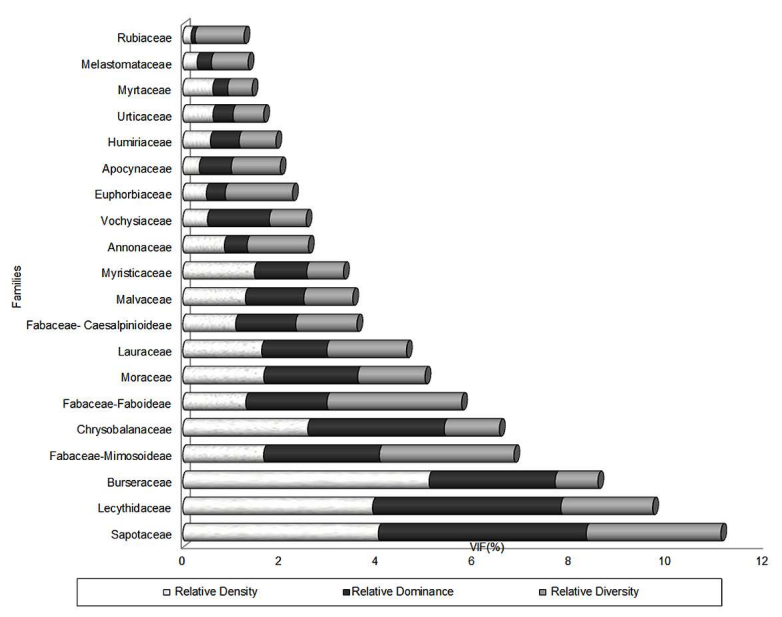

Figure 2 - Familiar importance value (VIF\%) for the 20 families with the highest values at the Experimental site of Embrapa Amazônia Ocidental, Manaus, Amazonas State, Brazil.

Figura 2 - Composição do Valor de Importância Familiar - VIF (\%) para as 20 famílias com os maiores valores, no Campo Experimental da Embrapa Amazônia Ocidental, Manaus, AM, Brasil.

Revista Árvore, Viçosa-MG, v.40, n.4, p.603-615, 2016

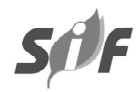


the three first families grouped approximately $39 \%$ of the total number of individuals. A great occurrence of rare species was found ( 1 individual/plot-1ha), whose values ranged from 30 to $42 \%$ of the total number of species recorded in the sampled plots. The families Fabaceae-Mimosoideae, FabaceaeFaboideae and Fabaceae-Caesalpinoideae, in a decreasing order, are the subfamilies that showed the largest number of species contributing to this value.

A great variability is found in the occurrence of species distributed in IV classes (\%) within the 15 sampled plots (Figure 3), especially in the two smallest classes that group values lower than $0.66 \%$. Those species correspond to approximately $83 \%$ of the species identified in the study area.

Plots 15, 150 and 273 have a larger number of exclusive species within the smallest class $(<0.096 \%)$ and as the IV values increase, a more even distribution of species in the 15 plots is found. Of the 85 species grouped in the smallest class of IV $(<0.096 \%), 41$ species appear with exclusive occurrence. These species have low density, with $87 \%$ of them occurring with only one (01) individual and $13 \%$ with two to six. Duckesia warty (Ducke) Cuatrec. (Humiriaceae), presents six individuals that were registered only in the plot 106 , which is on the low topographic position, containing higher content of moisture and with sandy texture. Considering the plots 15, 273 and 150 , these sum $39 \%$ of the number of species, with the lowest value of IV and with occurrence restricted to these plots. The fact of working with a large set of data, allows better observation of these variations, giving greater reliability to the observed results (Table 1).

The distribution of individuals in diameter classes in $10 \mathrm{~cm}$ of amplitude (Figure 4 ) shows a J-inverse distribution, characteristic of natural forests. The total amplitude of diameters ranged from 10 to 210 $\mathrm{cm}$, where it is observed the occurrence of individuals in almost all diameter classes, except for classes $140-180 \mathrm{~cm}$ and $190-200 \mathrm{~cm}$.

Most individuals (61\%) is distributed in the smallest diameter class $(10-20 \mathrm{~cm})$. The first two classes $(10-30 \mathrm{~cm})$ concentrate $80 \%$ of the total number of individuals in the 15 hectares sampled.

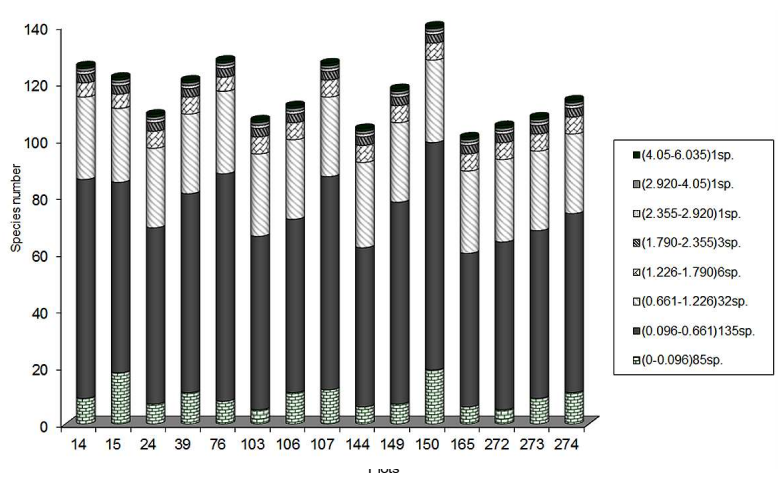

Figure 3 - Distribution of the number species within plots by class of VI (\%) for the 15 plots of $100 \times 100 \mathrm{~m}$, situated at the Experimental site of Embrapa Amazônia Ocidental, Manaus, Amazonas State, Brazil.

Figura 3 - Distribuição do número de espécies nas parcelas segundo as classes de VI (\%) para 15 parcelas de $100 \times 100$ m localizadas no Campo Experimental da Embrapa Amazônia Ocidental, Manaus-AM, Brasil.

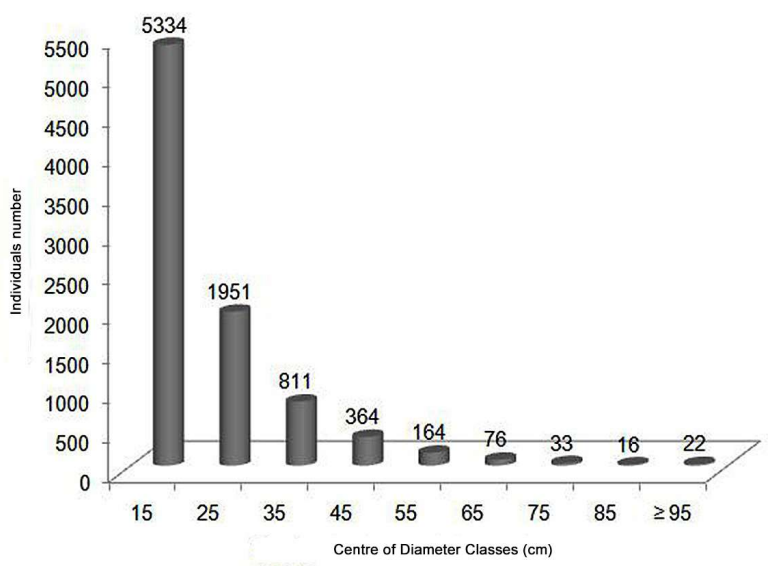

Figure 4 - Total number of individuals by classes of diameter of $10 \mathrm{~cm}$ range, at the Experimental site of Embrapa Amazônia Ocidental, Manaus, Amazonas State, Brazil.

Figura 4-Número total de indivíduos por classes de diâmetro de amplitude $10 \mathrm{~cm}$, no Campo Experimental da Embrapa Amazônia Ocidental, Manaus, AM, Brasil.

Buchenavia grandis Ducke (Combretaceae) and Andira parviflora Ducke (Fabaceae-Faboideae) occurred in diametric class 180-190 cm; Caryocar pallidum A.C. Sm. (Caryocaraceae) and Dinizia excelsa Ducke (Fabaceae-Mimosoideae), in the largest class $(200-210 \mathrm{~cm})$. 
Table 1 - Horizontal structure of 15 ha of permanente plots in a terra firme dense forest in Central Amazônia.

Tabela 1 - Estrutura horizontal de 15 ha de parcelas permanentes de uma floresta densa de terra firme na Amazônia Central.

\begin{tabular}{|c|c|c|c|c|c|c|c|}
\hline Family & Scientific name & $\mathrm{N}$ & DR & FR & DoR & VI & VI (\%) \\
\hline Burseraceae & Protium hebetatum Daly & 1037 & 11.82 & 0.87 & 5.42 & 18.10 & 6.03 \\
\hline Lecythidaceae & $\begin{array}{l}\text { Eschweilera coriacea (DC.) } \\
\text { S.A. Mori }\end{array}$ & 471 & 5.37 & 0.87 & 3.72 & 9.95 & 3.32 \\
\hline Chrysobalanaceae & Licania oblongifolia Standl. & 310 & 3.53 & 0.87 & 3.28 & 7.68 & 2.56 \\
\hline Sapotaceae & Pouteria minima T.D.Penn. & 293 & 3.34 & 0.87 & 2.85 & 7.06 & 2.35 \\
\hline Lauraceae & Ocotea cernua(Nees) Mez s.l. & 258 & 2.94 & 0.87 & 2.30 & 6.10 & 2.03 \\
\hline Malvaceae & Scleronema micranthum Ducke & 197 & 2.24 & 0.87 & 2.63 & 5.74 & 1.91 \\
\hline Chrysobalanaceae & Licania apelata (E.Mey.) Fritsch & 172 & 1.96 & 0.87 & 2.06 & 4.89 & 1.63 \\
\hline Lecythidaceae & Eschweilera collina Eyma & 176 & 2.01 & 0.87 & 1.98 & 4.85 & 1.62 \\
\hline Lecythidaceae & Couratari alta Kunth. & 125 & 1.42 & 0.87 & 1.97 & 4.26 & 1.42 \\
\hline Sapotaceae & $\begin{array}{l}\text { Chrysophyllum sanguinolentum } \\
\text { (Pierre) Baehni }\end{array}$ & 146 & 1.66 & 0.69 & 1.67 & 4.03 & 1.34 \\
\hline Moraceae & Naucleopsis caloneura (Huber) Ducke & 170 & 1.94 & 0.87 & 1.02 & 3.83 & 1.28 \\
\hline Moraceae & Brosimum rubescens Taub. & 106 & 1.21 & 0.87 & 1.73 & 3.81 & 1.27 \\
\hline Elaeocarpaceae & Sloanea excelsa Ducke & 92 & 1.05 & 0.87 & 1.63 & 3.54 & 1.18 \\
\hline Urticaceae & Pourouma myrmecophyla Ducke & 144 & 1.64 & 0.81 & 1.08 & 3.53 & 1.18 \\
\hline Fabaceae-Mimosoideae & Inga obidensis Ducke & 132 & 1.50 & 0.87 & 1.10 & 3.47 & 1.16 \\
\hline Myrtaceae & Psidium araca Raddi & 152 & 1.73 & 0.87 & 0.87 & 3.47 & 1.16 \\
\hline Myristicaceae & Iryanthera coriacea Ducke & 146 & 1.66 & 0.87 & 0.75 & 3.28 & 1.09 \\
\hline Lecythidaceae & Couratari stellata A.C.Sm. & 74 & 0.84 & 0.87 & 1.42 & 3.13 & 1.04 \\
\hline Myristicaceae & Virola michelii Heckel & 128 & 1.46 & 0.81 & 0.82 & 3.09 & 1.03 \\
\hline Chrysobalanaceae & Licania micrantha Miq. & 86 & 0.98 & 0.87 & 1.21 & 3.06 & 1.02 \\
\hline Combretaceae & Buchenavia grandis Ducke & 31 & 0.35 & 0.75 & 1.92 & 3.03 & 1.01 \\
\hline Humiriaceae & Vantanea macrocarpa Ducke & 93 & 1.06 & 0.87 & 1.05 & 2.97 & 0.99 \\
\hline Sapotaceae & Pouteria laurifolia (Gomes) Radlk & 128 & 1.46 & 0.69 & 0.77 & 2.93 & 0.98 \\
\hline Sapotaceae & Micropholis trunciflora Ducke & 89 & 1.01 & 0.81 & 1.05 & 2.87 & 0.96 \\
\hline Vochysiaceae & Qualea albiflora Warm & 49 & 0.56 & 0.75 & 1.53 & 2.84 & 0.95 \\
\hline $\begin{array}{l}\text { Fabaceae- } \\
\text { Caesalpinioideae }\end{array}$ & Sclerolobium helanocarpus Ducke & 75 & 0.85 & 0.75 & 1.16 & 2.77 & 0.92 \\
\hline Myristicaceae & $\begin{array}{l}\text { Osteophloeum platyspermum } \\
\text { (A.DC.) Warb. }\end{array}$ & 54 & 0.62 & 0.69 & 1.38 & 2.69 & 0.90 \\
\hline Sapotaceae & Glycoxylon pedicellatum (Ducke) Ducke & 90 & 1.03 & 0.87 & 0.77 & 2.66 & 0.89 \\
\hline Sapotaceae & $\begin{array}{l}\text { Pouteria venosa (Mart.) } \\
\text { Baehni ssp. amazonica T.D.Penn. }\end{array}$ & 74 & 0.84 & 0.75 & 1.05 & 2.64 & 0.88 \\
\hline Goupiaceae & Goupia glabra Aubl. & 32 & 0.36 & 0.58 & 1.69 & 2.64 & 0.88 \\
\hline Burseraceae & Protium pilosissimum Engl. & 126 & 1.44 & 0.52 & 0.59 & 2.55 & 0.85 \\
\hline Burseraceae & Trattinnickia burserifolia Mart. & 60 & 0.68 & 0.81 & 1.03 & 2.52 & 0.84 \\
\hline Fabaceae-Faboideae & Bocoa viridiflora (Ducke) R.S.Cowan & 85 & 0.97 & 0.75 & 0.75 & 2.47 & 0.82 \\
\hline Apocynaceae & Aspidosperma marcgravianum Woodson & 36 & 0.41 & 0.81 & 1.19 & 2.41 & 0.80 \\
\hline $\begin{array}{l}\text { Fabaceae- } \\
\text { Caesalpinioideae }\end{array}$ & Eperua duckeana R.S.Cowan & 72 & 0.82 & 0.46 & 1.04 & 2.32 & 0.77 \\
\hline Olacaceae & Minquartia guianensis Aubl. & 75 & 0.85 & 0.64 & 0.80 & 2.29 & 0.76 \\
\hline Burseraceae & $\begin{array}{l}\text { Protium heptaphyllum (Aubl.) } \\
\text { ssp. ulei (Swart) Daly }\end{array}$ & 82 & 0.93 & 0.81 & 0.49 & 2.24 & 0.75 \\
\hline Annonaceae & Unonopsis duckei (R.\&P.)Macba. & 83 & 0.95 & 0.81 & 0.37 & 2.13 & 0.71 \\
\hline $\begin{array}{l}\text { Fabaceae- } \\
\text { Caesalpinioideae }\end{array}$ & Eperua glabriflora (Ducke) R.S.Cowan & 50 & 0.57 & 0.87 & 0.68 & 2.11 & 0.70 \\
\hline Sapotaceae & $\begin{array}{l}\text { Micropholis guyanensis (A.DC.) } \\
\text { Pierre ssp.duckeana (Baehni) }\end{array}$ & 60 & 0.68 & 0.69 & 0.73 & 2.11 & 0.70 \\
\hline Vochysiaceae & Erisma bicolor Ducke & 37 & 0.42 & 0.81 & 0.84 & 2.07 & 0.69 \\
\hline Caryocaraceae & Caryocar pallidum A.C. Smith & 12 & 0.14 & 0.52 & 1.40 & 2.06 & 0.69 \\
\hline Nyctaginaceae & Neea oppositifolia Ruiz \& Pav. & 69 & 0.79 & 0.81 & 0.45 & 2.05 & 0.68 \\
\hline Chrysobalanaceae & Licania heteromorpha Benth. & 59 & 0.67 & 0.69 & 0.62 & 1.99 & 0.66 \\
\hline
\end{tabular}


Table 1...

Tabela 1...

\begin{tabular}{|c|c|c|c|c|c|c|c|}
\hline Sapotaceae & $\begin{array}{l}\text { Manilkara bidentata } \\
\text { (A.DC.) A. Chev. }\end{array}$ & 39 & 0.44 & 0.64 & 0.89 & 1.97 & 0.66 \\
\hline Fabaceae-Mimosoideae & $\begin{array}{l}\text { Zigia racemosa } \\
\text { (Ducke) Barneby \& J.W. Grimes }\end{array}$ & 44 & 0.50 & 0.75 & 0.70 & 1.95 & 0.65 \\
\hline Moraceae & Clarisia racemosa Ruiz \& Pav. & 29 & 0.33 & 0.69 & 0.91 & 1.93 & 0.64 \\
\hline Lecythidaceae & Lecythis barnebyi Mori & 31 & 0.35 & 0.64 & 0.88 & 1.87 & 0.62 \\
\hline Melastomataceae & Mouriri callocarpa Ducke & 37 & 0.42 & 0.87 & 0.57 & 1.86 & 0.62 \\
\hline Sapotaceae & $\begin{array}{l}\text { Pouteria caimito } \\
\text { (Ruiz \& Pav.) Radlk. }\end{array}$ & 41 & 0.47 & 0.75 & 0.59 & 1.81 & 0.60 \\
\hline Chrysobalanaceae & Licania laevigata Prance & 26 & 0.30 & 0.58 & 0.93 & 1.81 & 0.60 \\
\hline Moraceae & $\begin{array}{l}\text { Brosimum lactescens } \\
\text { (S.Moore) C.C.Berg. }\end{array}$ & 24 & 0.27 & 0.46 & 1.06 & 1.79 & 0.60 \\
\hline $\begin{array}{l}\text { Fabaceae- } \\
\text { Caesalpinioideae }\end{array}$ & Tachigali cf. myrmecophila Ducke & 42 & 0.48 & 0.87 & 0.44 & 1.79 & 0.60 \\
\hline Lecythidaceae & $\begin{array}{c}\text { Eschweilera atropetiolata } \\
\text { S.A.Mori }\end{array}$ & 47 & 0.54 & 0.75 & 0.50 & 1.78 & 0.59 \\
\hline Fabaceae-Mimosoideae & $\begin{array}{c}\text { Enterolobium } \\
\text { schomburgkii Benth. }\end{array}$ & 33 & 0.38 & 0.69 & 0.71 & 1.78 & 0.59 \\
\hline Annonaceae & $\begin{array}{l}\text { Bocageopsis multiflora } \\
\text { (Mart.) R.E.Fr. }\end{array}$ & 45 & 0.51 & 0.81 & 0.45 & 1.77 & 0.59 \\
\hline Fabaceae-Faboideae & Swartzia recurva Poepp. & 47 & 0.54 & 0.64 & 0.54 & 1.71 & 0.57 \\
\hline Myristicaceae & Virola calophylla Warb. & 57 & 0.65 & 0.69 & 0.28 & 1.62 & 0.54 \\
\hline Annonaceae & Guatterria olivacea R.R.Fr. & 45 & 0.51 & 0.81 & 0.27 & 1.59 & 0.53 \\
\hline Fabaceae-Faboideae & Andira trifoliata S.A.Mori & 50 & 0.57 & 0.46 & 0.56 & 1.59 & 0.53 \\
\hline Sapotaceae & Ecclinusa guianensis Eyma & 30 & 0.34 & 0.46 & 0.77 & 1.57 & 0.52 \\
\hline Fabaceae-Faboideae & $\begin{array}{c}\text { Swartzia schomburgkii Benth. } \\
\text { Var. guyanensis R.S. Cowan }\end{array}$ & 20 & 0.23 & 0.52 & 0.76 & 1.51 & 0.50 \\
\hline Euphorbiaceae & Hevea guianensis Aubl. & 47 & 0.54 & 0.40 & 0.56 & 1.50 & 0.50 \\
\hline Lecythidaceae & Lecythis graciena S.A.Mori & 34 & 0.39 & 0.64 & 0.46 & 1.49 & 0.50 \\
\hline Fabaceae-Mimosoideae & Dinizia excelsa Ducke & 3 & 0.03 & 0.17 & 1.23 & 1.44 & 0.48 \\
\hline Malvaceae & Theobroma sylvestre Mart. & 53 & 0.60 & 0.69 & 0.14 & 1.44 & 0.48 \\
\hline Opiliaceae & Agonandra brasiliensis Miers & 20 & 0.23 & 0.58 & 0.62 & 1.43 & 0.48 \\
\hline Apocynaceae & $\begin{array}{c}\text { Geissospermum argenteum } \\
\text { Woodson }\end{array}$ & 23 & 0.26 & 0.64 & 0.48 & 1.38 & 0.46 \\
\hline Fabaceae-Faboideae & Andira parviflora Ducke & 14 & 0.16 & 0.46 & 0.75 & 1.37 & 0.46 \\
\hline Meliaceae & Trichilia micropetala T.D.Penn. & 40 & 0.46 & 0.69 & 0.21 & 1.36 & 0.45 \\
\hline Fabaceae-Mimosoideae & Inga alba $(\mathrm{Sw}) \mathrm{Willd}$ & 44 & 0.50 & 0.58 & 0.28 & 1.35 & 0.45 \\
\hline Dichapetalaceae & $\begin{array}{l}\text { Tapura amazonica } \\
\text { Poepp. \& Endl. }\end{array}$ & 45 & 0.51 & 0.58 & 0.26 & 1.35 & 0.45 \\
\hline Fabaceae-Mimosoideae & Piptadenia suavolens Miq. & 18 & 0.21 & 0.52 & 0.60 & 1.33 & 0.44 \\
\hline Clusiaceae & $\begin{array}{c}\text { Distomovita brasiliensis } \\
\text { D’Arcy }\end{array}$ & 37 & 0.42 & 0.69 & 0.20 & 1.31 & 0.44 \\
\hline Sapindaceaea & Talisia cf. cupularis Radlk. & 41 & 0.47 & 0.64 & 0.20 & 1.30 & 0.43 \\
\hline Moraceae & Helicostylis scabra (Macbr.) & 32 & 0.36 & 0.69 & 0.24 & 1.30 & 0.43 \\
\hline Lauraceae & Licaria guianensis Aubl. & 33 & 0.38 & 0.64 & 0.28 & 1.29 & 0.43 \\
\hline Simaroubaceae & Simarouba amara Aubl. & 21 & 0.24 & 0.58 & 0.44 & 1.26 & 0.42 \\
\hline Lecythidaceae & Gustavia elliptica S.A.Mori & 37 & 0.42 & 0.69 & 0.13 & 1.25 & 0.42 \\
\hline Lauraceae & Aniba hostmaniana (Ness) Mez. & 37 & 0.42 & 0.64 & 0.17 & 1.22 & 0.41 \\
\hline Humiriaceae & Endopleura uchi (Huber) Cuatrec. & 21 & 0.24 & 0.58 & 0.41 & 1.22 & 0.41 \\
\hline Moraceae & Helianthostylis sprucei Baill. & 44 & 0.50 & 0.58 & 0.14 & 1.22 & 0.41 \\
\hline Anacardiaceae & Anacardium parvifolium Ducke & 16 & 0.18 & 0.46 & 0.55 & 1.20 & 0.40 \\
\hline Fabaceae-Mimosoideae & Inga gracilifolia Ducke & 34 & 0.39 & 0.64 & 0.16 & 1.19 & 0.40 \\
\hline Sapotaceae & Pouteria reticulata (Engl.) Eyma & 13 & 0.15 & 0.40 & 0.59 & 1.15 & 0.38 \\
\hline Olacaceae & Pytchopetalum olacoides Benth. & 29 & 0.33 & 0.52 & 0.27 & 1.12 & 0.37 \\
\hline Vochysiaceae & Ruizterania cassiquiarensis & 18 & 0.21 & 0.52 & 0.37 & 1.10 & 0.37 \\
\hline
\end{tabular}


Table 1...

Tabela 1..

\begin{tabular}{|c|c|c|c|c|c|c|c|}
\hline Malvaceae & $\begin{array}{l}\text { (Spruce ex Warm.) } \\
\text { Lueheopsis rosea } \\
\text { (Ducke) Burret }\end{array}$ & 22 & 0.25 & 0.52 & 0.32 & 1.09 & 0.36 \\
\hline Fabaceae-Mimosoideae & Stryphnodendron sp. & 26 & 0.30 & 0.58 & 0.21 & 1.08 & 0.36 \\
\hline Fabaceae-Mimosoideae & Parkia pendula (Willd.) Walp. & 4 & 0.05 & 0.23 & 0.80 & 1.08 & 0.36 \\
\hline Lecythidaceae & Lecythis usidata Camb. & 18 & 0.21 & 0.64 & 0.24 & 1.08 & 0.36 \\
\hline $\begin{array}{l}\text { Fabaceae- } \\
\text { Caesalpinioideae }\end{array}$ & $\begin{array}{l}\text { Macrolobium limbatum } \\
\text { Spruce ex Benth }\end{array}$ & 33 & 0.38 & 0.46 & 0.22 & 1.06 & 0.35 \\
\hline Lauraceae & Sextonia rubra (Mez) Van der Werff & 13 & 0.15 & 0.46 & 0.45 & 1.06 & 0.35 \\
\hline Lauraceae & $\begin{array}{c}\text { Mezilaurus itauba (Meissn.) } \\
\text { Taubert ex Mez }\end{array}$ & 20 & 0.23 & 0.58 & 0.24 & 1.05 & 0.35 \\
\hline Vochysiaceae & Vochysia vismiaefolia Spruce ex Warm. & 13 & 0.15 & 0.40 & 0.49 & 1.04 & 0.35 \\
\hline Malvaceae & Sterculia pruriens (Aubl.) K.Schum. & 25 & 0.28 & 0.52 & 0.22 & 1.03 & 0.34 \\
\hline Fabaceae-Faboideae & Swartzia corrugata Benth. & 26 & 0.30 & 0.52 & 0.19 & 1.01 & 0.34 \\
\hline Sapotaceae & Pouteria oblanceolata Pires & 17 & 0.19 & 0.46 & 0.30 & 0.95 & 0.32 \\
\hline Euphorbiaceae & Conceveiba guianensis Aubl. & 24 & 0.27 & 0.46 & 0.21 & 0.95 & 0.32 \\
\hline Lauraceae & Licaria $\mathrm{sp}$ & 20 & 0.23 & 0.58 & 0.13 & 0.93 & 0.31 \\
\hline Fabaceae-Mimosoideae & Inga stipularis DC. & 19 & 0.22 & 0.46 & 0.24 & 0.92 & 0.31 \\
\hline Moraceae & $\begin{array}{l}\text { Brosimum utile (H.B.K.) Pittier ssp. } \\
\text { ovatifolium (Ducke) C.C.Berg. }\end{array}$ & 15 & 0.17 & 0.40 & 0.33 & 0.90 & 0.30 \\
\hline Violaceae & Rinorea racemosa (Mart.) Kuntze & 26 & 0.30 & 0.52 & 0.06 & 0.88 & 0.29 \\
\hline Annonaceae & Xylopia calophylla R.E. Fr. & 21 & 0.24 & 0.52 & 0.10 & 0.86 & 0.29 \\
\hline Fabaceae-Mimosoideae & Parkia multijuga Benth. & 13 & 0.15 & 0.40 & 0.30 & 0.85 & 0.28 \\
\hline Bignoniaceae & Jacaranda copaia (Aubl.) D. Don. & 22 & 0.25 & 0.46 & 0.13 & 0.84 & 0.28 \\
\hline Simaroubaceae & $\begin{array}{c}\text { Simaba polyphylla (Cavalcante) } \\
\text { W.Thomas }\end{array}$ & 21 & 0.24 & 0.52 & 0.08 & 0.84 & 0.28 \\
\hline Rubiaceae & $\begin{array}{l}\text { Duroia fusifera Hook. } \\
\text { F. ex K. Schum. }\end{array}$ & 18 & 0.21 & 0.52 & 0.11 & 0.84 & 0.28 \\
\hline Chrysobalanaceae & Couepia longipendula Pilg. & 19 & 0.22 & 0.35 & 0.25 & 0.81 & 0.27 \\
\hline Melastomataceae & Tococa guianensis Aubl. & 17 & 0.19 & 0.52 & 0.09 & 0.80 & 0.27 \\
\hline Fabaceae-Faboideae & Dipteryx magnifica Ducke & 12 & 0.14 & 0.40 & 0.25 & 0.79 & 0.26 \\
\hline Fabaceae-Mimosoideae & $\begin{array}{c}\text { Abarema jupunba (Willd.) } \\
\text { Britton \&Killip }\end{array}$ & 21 & 0.24 & 0.29 & 0.26 & 0.79 & 0.26 \\
\hline Fabaceae-Faboideae & Diplotropis rodriguesii Lima & 18 & 0.21 & 0.35 & 0.22 & 0.77 & 0.26 \\
\hline Fabaceae-Faboideae & Paramachaerium ormosioides Ducke & 13 & 0.15 & 0.52 & 0.09 & 0.76 & 0.25 \\
\hline Malvaceae & Apeiba echinata Gaertner & 10 & 0.11 & 0.52 & 0.12 & 0.75 & 0.25 \\
\hline Vochysiaceae & Qualea paraensis Ducke & 9 & 0.10 & 0.23 & 0.41 & 0.74 & 0.25 \\
\hline Fabaceae-Faboideae & Swartzia reticulata Ducke & 13 & 0.15 & 0.40 & 0.18 & 0.74 & 0.25 \\
\hline Icacinaceae & $\begin{array}{l}\text { Emmotum aff. nitens Benth. } \\
\text { Ex Miers }\end{array}$ & 15 & 0.17 & 0.46 & 0.10 & 0.73 & 0.24 \\
\hline Burseraceae & Protium rubrum Cuatrec. & 27 & 0.31 & 0.29 & 0.14 & 0.73 & 0.24 \\
\hline Clusiaceae & Platonia insignis Mart. & 11 & 0.13 & 0.40 & 0.20 & 0.73 & 0.24 \\
\hline Solanaceae & Duckeodendron cestoides Kuhlm. & 6 & 0.07 & 0.29 & 0.37 & 0.72 & 0.24 \\
\hline Fabaceae-Mimosoideae & Inga suberosa T.D.Penn. & 12 & 0.14 & 0.52 & 0.05 & 0.71 & 0.24 \\
\hline Euphorbiaceae & Micranda siphonoides Benth. & 13 & 0.15 & 0.46 & 0.05 & 0.67 & 0.22 \\
\hline Malvaceae & Theobroma subincanum Mart. & 15 & 0.17 & 0.40 & 0.08 & 0.66 & 0.22 \\
\hline Apocynaceae & Ambelania duckei Markgr. & 12 & 0.14 & 0.46 & 0.05 & 0.65 & 0.22 \\
\hline Malvaceae & $\begin{array}{l}\text { Pseudobombax munguba } \\
\text { (Mart. \& Zucc) Dugand }\end{array}$ & 17 & 0.19 & 0.35 & 0.11 & 0.65 & 0.22 \\
\hline Melastomataceae & Miconia reglelii Cogn. & 9 & 0.10 & 0.46 & 0.06 & 0.62 & 0.21 \\
\hline Meliaceae & Carapa guianensis Aubl. & 23 & 0.26 & 0.06 & 0.29 & 0.61 & 0.20 \\
\hline Fabaceae- Caesalpinioideae & Peltogyne paniculata Benth. & 8 & 0.09 & 0.40 & 0.11 & 0.61 & 0.20 \\
\hline Erythroxylaceae & Erythroxylum amplum Bth. & 15 & 0.17 & 0.35 & 0.08 & 0.60 & 0.20 \\
\hline
\end{tabular}


Table 1...

Tabela 1...

\begin{tabular}{|c|c|c|c|c|c|c|c|}
\hline Meliaceae & Trichilia septentrionales C.DC. & 10 & 0.11 & 0.40 & 0.08 & $0 . \overline{59}$ & 0.20 \\
\hline Vochysiaceae & Qualea acuminata Aubl. & 8 & 0.09 & 0.29 & 0.20 & 0.58 & 0.19 \\
\hline Sapotaceae & Manilkara amazonica (Huber) Stand. & 11 & 0.13 & 0.29 & 0.16 & 0.57 & 0.19 \\
\hline Bixaceae & Bixa orellana $\mathrm{L}$ & 15 & 0.17 & 0.35 & 0.05 & 0.57 & 0.19 \\
\hline Lauraceae & Licania $\mathrm{sp}$ & 11 & 0.13 & 0.29 & 0.15 & 0.57 & 0.19 \\
\hline Fabaceae-Mimosoideae & Parkia nitida Miq. & 9 & 0.10 & 0.35 & 0.11 & 0.56 & 0.19 \\
\hline Fabaceae-Mimosoideae & Inga cordatoalata Ducke & 14 & 0.16 & 0.35 & 0.05 & 0.56 & 0.19 \\
\hline Humiriaceae & Vantanea guiannensis (Aubl.) Ducke & 10 & 0.11 & 0.35 & 0.09 & 0.56 & 0.19 \\
\hline Urticaceae & Pouroma guianensis Aubl. & 12 & 0.14 & 0.35 & 0.07 & 0.55 & 0.18 \\
\hline Lauraceae & Ocotea cymbarum H.B.K. & 11 & 0.13 & 0.29 & 0.12 & 0.53 & 0.18 \\
\hline Humiriaceae & Vantanea micrantha Ducke & 11 & 0.13 & 0.29 & 0.11 & 0.53 & 0.18 \\
\hline Bignoniaceae & Tabebuia serratifolia (Vahl) Nichols & 6 & 0.07 & 0.35 & 0.11 & 0.52 & 0.17 \\
\hline Annonaceae & Pseudoxandra coriacea R.E.Fr. & 9 & 0.10 & 0.35 & 0.07 & 0.52 & 0.17 \\
\hline Anacardiaceae & Astronium lecointei Ducke & 7 & 0.08 & 0.29 & 0.14 & 0.51 & 0.17 \\
\hline Melastomataceae & Mouriri angulicosta Morley & 7 & 0.08 & 0.29 & 0.10 & 0.47 & 0.16 \\
\hline Myrtaceae & Myrcia Paivae O. Berg & 8 & 0.09 & 0.35 & 0.03 & 0.47 & 0.16 \\
\hline Annonaceae & Annona ambotay Aubl. & 12 & 0.14 & 0.23 & 0.10 & 0.47 & 0.16 \\
\hline Humiriaceae & Sacoglotis matogrossensis Aubl. & 9 & 0.10 & 0.29 & 0.07 & 0.46 & 0.15 \\
\hline Rubiaceae & Chimarrhis sp. & 7 & 0.08 & 0.35 & 0.03 & 0.46 & 0.15 \\
\hline Peraceae & $\begin{array}{c}\text { Pogonophora schomburgkiana } \\
\text { Miers ex Benth. }\end{array}$ & 7 & 0.08 & 0.29 & 0.08 & 0.45 & 0.15 \\
\hline Fabaceae-Faboideae & Dipteryx odorata (Aubl.) Willd. & 6 & 0.07 & 0.29 & 0.08 & 0.44 & 0.15 \\
\hline Moraceae & Brosimum parinarioides Ducke & 6 & 0.07 & 0.12 & 0.24 & 0.43 & 0.14 \\
\hline Melastomataceae & Miconia elaeagnoides Cogn. & 7 & 0.08 & 0.29 & 0.05 & 0.42 & 0.14 \\
\hline Fabaceae-Mimosoideae & Pithecellobium elegans Ducke & 6 & 0.07 & 0.12 & 0.24 & 0.42 & 0.14 \\
\hline Fabaceae-Faboideae & Swartzia ulei Harms & 12 & 0.14 & 0.17 & 0.10 & 0.41 & 0.14 \\
\hline Sapotaceae & $\begin{array}{c}\text { Pouteria ambelaniifolia (Sandwith) } \\
\text { T.D.Penn }\end{array}$ & 5 & 0.06 & 0.23 & 0.12 & 0.41 & 0.14 \\
\hline Sapotaceae & $\begin{array}{c}\text { Chrysophyllum sparsiflorum } \\
\text { Klotzsch ex Miq. }\end{array}$ & 5 & 0.06 & 0.23 & 0.11 & 0.39 & 0.13 \\
\hline Lauraceae & Licaria canella (Meissn.) Kosterm. & 7 & 0.08 & 0.23 & 0.08 & 0.39 & 0.13 \\
\hline Euphorbiaceae & Pausandra macropetala Ducke & 12 & 0.14 & 0.17 & 0.07 & 0.38 & 0.13 \\
\hline Euphorbiaceae & Mabea subsessilis Pax \& K. Hoffm. & 10 & 0.11 & 0.23 & 0.03 & 0.38 & 0.13 \\
\hline Lecythidaceae & Cariniana $\mathrm{sp}$ & 3 & 0.03 & 0.17 & 0.17 & 0.37 & 0.12 \\
\hline Apocynaceae & Couma utiles Mart. & 5 & 0.06 & 0.23 & 0.08 & 0.37 & 0.12 \\
\hline Burseraceae & Protium divaricatum Engl. & 4 & 0.05 & 0.17 & 0.12 & 0.34 & 0.11 \\
\hline Lauraceae & Ocotea $\mathrm{sp}$ & 8 & 0.09 & 0.17 & 0.07 & 0.33 & 0.11 \\
\hline Salicaceae & Casearia grandiflora Cambess & 6 & 0.07 & 0.23 & 0.03 & 0.33 & 0.11 \\
\hline Monimiaceae & Siparuna sp. & 6 & 0.07 & 0.23 & 0.02 & 0.32 & 0.11 \\
\hline Quiinaceae & Touroulia guianensis Aubl. & 6 & 0.07 & 0.23 & 0.02 & 0.32 & 0.11 \\
\hline Lauraceae & Aniba megaphylla Mez & 5 & 0.06 & 0.23 & 0.03 & 0.32 & 0.11 \\
\hline Fabaceae- Caesalpinioideae & Hymenea parvifolia Huber & 4 & 0.05 & 0.23 & 0.04 & 0.31 & 0.10 \\
\hline Combretaceae & Terminalia dichotoma G.Meyer & 4 & 0.05 & 0.23 & 0.04 & 0.31 & 0.10 \\
\hline Euphorbiaceae & Croton lanjouwensis Jabl. & 5 & 0.06 & 0.17 & 0.08 & 0.31 & 0.10 \\
\hline Fabaceae-Faboideae & Dipteryx polyphylla Huber & 4 & 0.05 & 0.17 & 0.09 & 0.31 & 0.10 \\
\hline Apocynaceae & Aspidosperma album (Vahl.) R. Bem. & 4 & 0.05 & 0.17 & 0.09 & 0.31 & 0.10 \\
\hline Lecythidaceae & Lecythis prancei S.A. Mori & 4 & 0.05 & 0.23 & 0.03 & 0.31 & 0.10 \\
\hline Rubiaceae & Palicourea corymbifera Mull. Arg. & 9 & 0.10 & 0.17 & 0.03 & 0.31 & 0.10 \\
\hline Moraceae & Sorocea guilleminiana Gaudich. & 9 & 0.10 & 0.17 & 0.03 & 0.31 & 0.10 \\
\hline Fabaceae-Faboideae & Dipteryx $\mathrm{sp}$ & 4 & 0.05 & 0.06 & 0.20 & 0.30 & 0.10 \\
\hline Sapotaceae & Poteuria platyphylla (A.C.Sm.) Baehni & 3 & 0.03 & 0.17 & 0.09 & 0.30 & 0.10 \\
\hline Sapotaceae & Micropholis sp. & 3 & 0.03 & 0.17 & 0.08 & 0.29 & 0.10 \\
\hline Lecythidaceae & Lecythis poiteaui Berg. & 6 & 0.07 & 0.12 & 0.10 & 0.29 & 0.10 \\
\hline
\end{tabular}

Revista Árvore, Viçosa-MG, v.40, n.4, p.603-615, 2016 
Table 1...

Tabela 1...

\begin{tabular}{|c|c|c|c|c|c|c|c|}
\hline Clusiaceae & Symphonia globulifera L. & 7 & 0.08 & 0.12 & 0.09 & 0.28 & 0.09 \\
\hline Sapotaceae & Pouteria petiolata T.D. Penn. & 4 & 0.05 & 0.17 & 0.06 & 0.28 & 0.09 \\
\hline Euphorbiaceae & Conceveiba martiana Baill & 5 & 0.06 & 0.12 & 0.10 & 0.27 & 0.09 \\
\hline Urticaceae & Cecropia sciadophylla Mart. & 5 & 0.06 & 0.17 & 0.04 & 0.27 & 0.09 \\
\hline Fabaceae-Faboideae & Platymiscium duckei Huber & 4 & 0.05 & 0.17 & 0.05 & 0.27 & 0.09 \\
\hline Euphorbiaceae & Mabea sp. & 6 & 0.07 & 0.17 & 0.01 & 0.26 & 0.09 \\
\hline Chrysobalanaceae & Couepia bracteosa Benth. & 4 & 0.05 & 0.12 & 0.09 & 0.26 & 0.09 \\
\hline Clusiaceae & Vismia japurensis Reichardt & 4 & 0.05 & 0.17 & 0.04 & 0.26 & 0.09 \\
\hline Violaceae & Rinorea guianensis Aubl. & 5 & 0.06 & 0.17 & 0.02 & 0.25 & 0.08 \\
\hline Apocynaceae & Couma macrocarpa Barb. & 4 & 0.05 & 0.17 & 0.03 & 0.25 & 0.08 \\
\hline Moraceae & $\begin{array}{l}\text { Brosimum acutifolium Huber ssp. } \\
\text { Interjectum C.C.Berg }\end{array}$ & 3 & 0.03 & 0.12 & 0.10 & 0.25 & 0.08 \\
\hline Sapotaceae & Pradosia cochlearia(Lecomte) T.D.Penn & 3 & 0.03 & 0.17 & 0.04 & 0.25 & 0.08 \\
\hline Monimiaceae & Siparuna guianensis Aubl. & 4 & 0.05 & 0.17 & 0.03 & 0.24 & 0.08 \\
\hline Sapotaceae & Pouteria peruviensis (Aubrév.) Bernardi & 3 & 0.03 & 0.12 & 0.09 & 0.24 & 0.08 \\
\hline Ulmaceae & Ampelocera edentula Rusby & 4 & 0.05 & 0.17 & 0.02 & 0.24 & 0.08 \\
\hline Apocynaceae & Hymatanthus sucuba (spruce)Woodson & 3 & 0.03 & 0.17 & 0.03 & 0.24 & 0.08 \\
\hline Annonaceae & Guatteria sp. & 4 & 0.05 & 0.17 & 0.01 & 0.23 & 0.08 \\
\hline Euphorbiaceae & Glycydendron amazonicum Ducke & 3 & 0.03 & 0.17 & 0.02 & 0.23 & 0.08 \\
\hline Fabaceae-Faboideae & Swartzia tomentifera Harms & 6 & 0.07 & 0.12 & 0.04 & 0.23 & 0.08 \\
\hline Annonaceae & Xylopia amazonica R.E. Fr. & 3 & 0.03 & 0.17 & 0.01 & 0.21 & 0.07 \\
\hline Fabaceae-Faboideae & Ormosia grossa Rudd & 2 & 0.02 & 0.12 & 0.08 & 0.21 & 0.07 \\
\hline Putranjivaceae & Drypetes variabilis Uittien & 6 & 0.07 & 0.12 & 0.02 & 0.21 & 0.07 \\
\hline Anisophylleacea & $\begin{array}{l}\text { Anisophyllea manausensis } \\
\text { Pires \& W.A.Rodrigues }\end{array}$ & 3 & 0.03 & 0.12 & 0.05 & 0.20 & 0.07 \\
\hline Caryocaraceae & Caryocar villosum (Aubl.) Pers. & 2 & 0.02 & 0.12 & 0.06 & 0.20 & 0.07 \\
\hline Rubiaceae & Warszewiczia schwackei K.Schum. & 3 & 0.03 & 0.12 & 0.05 & 0.20 & 0.07 \\
\hline Humiriaceae & Duckesia verrucosa (Ducke) Cuatr. & 6 & 0.07 & 0.06 & 0.06 & 0.18 & 0.06 \\
\hline Peraceae & Pera heteranthera (Schrank) I.M. Johnst. & 2 & 0.02 & 0.12 & 0.04 & 0.18 & 0.06 \\
\hline Lecythidaceae & Corythophora rimosa W.A.Rodrigues & 2 & 0.02 & 0.12 & 0.04 & 0.18 & 0.06 \\
\hline Linaceae & Roucheria punctata Ducke & 3 & 0.03 & 0.12 & 0.03 & 0.18 & 0.06 \\
\hline Sapotaceae & Pouteria guianensis Aubl. & 4 & 0.05 & 0.06 & 0.07 & 0.17 & 0.06 \\
\hline Lauraceae & Aniba canellila (H.B.K.)Mez & 3 & 0.03 & 0.12 & 0.02 & 0.17 & 0.06 \\
\hline Icacinaceae & Emmotum acuminatum (Benth.) Miers & 2 & 0.02 & 0.12 & 0.03 & 0.17 & 0.06 \\
\hline Anacardiaceae & Anacardium spruceanum Engl. & 1 & 0.01 & 0.06 & 0.10 & 0.17 & 0.06 \\
\hline Lauraceae & Aniba rosaeodora Ducke & 3 & 0.03 & 0.12 & 0.01 & 0.16 & 0.05 \\
\hline Rubiaceae & Chimarrhis duckeana del Prete & 3 & 0.03 & 0.12 & 0.01 & 0.16 & 0.05 \\
\hline Rubiaceae & $\begin{array}{c}\text { Duroia saccifera (Mart.) Hook. } \\
\text { F. ex K. Schum. }\end{array}$ & 3 & 0.03 & 0.12 & 0.01 & 0.16 & 0.05 \\
\hline Apocynaceae & Couma sp. & 2 & 0.02 & 0.12 & 0.02 & 0.16 & 0.05 \\
\hline Moraceae & Brosimum potabile Ducke & 2 & 0.02 & 0.12 & 0.01 & 0.15 & 0.05 \\
\hline Burseraceae & Protium subserratum Engler & 2 & 0.02 & 0.12 & 0.01 & 0.15 & 0.05 \\
\hline Annonaceae & Xylopia brasiliensis Spreng. & 2 & 0.02 & 0.12 & 0.01 & 0.15 & 0.05 \\
\hline Myrtaceae & Myrcia magna Legrand & 2 & 0.02 & 0.12 & 0.01 & 0.15 & 0.05 \\
\hline Myristicaceae & Iryanthera juruensis Warb. & 2 & 0.02 & 0.12 & 0.01 & 0.15 & 0.05 \\
\hline Fabaceae-Faboideae & Swartzia cuspidata Spruce ex Benth. & 2 & 0.02 & 0.12 & 0.01 & 0.15 & 0.05 \\
\hline Myristicaceae & Virola caducifolia W.A. Rodrigues & 2 & 0.02 & 0.12 & 0.01 & 0.15 & 0.05 \\
\hline Fabaceae-Mimosoideae & Abarema $\mathrm{sp}$ & 2 & 0.02 & 0.12 & 0.00 & 0.14 & 0.05 \\
\hline Chrysobalanaceae & Licania adolphoduckei Prance & 2 & 0.02 & 0.12 & 0.00 & 0.14 & 0.05 \\
\hline Fabaceae-Faboideae & Dipteryx punctata (Blake) Amshoff & 1 & 0.01 & 0.06 & 0.07 & 0.13 & 0.04 \\
\hline Fabaceae-Mimosoideae & $\begin{array}{c}\text { Stryphnodendron pulcherrimum } \\
\text { (Willd.) Hochr. }\end{array}$ & 1 & 0.01 & 0.06 & 0.05 & 0.12 & 0.04 \\
\hline Urticaceae & Cecropia concolor Willd. & 1 & 0.01 & 0.06 & 0.05 & 0.12 & 0.04 \\
\hline
\end{tabular}


Table 1...

Tabela 1...

\begin{tabular}{|c|c|c|c|c|c|c|c|}
\hline Lecythidaceae & Couratari guianensis Aubl. & 2 & 0.02 & 0.06 & 0.03 & 0.11 & 0.04 \\
\hline Fabaceae-Mimosoideae & Parkia decussata Ducke & 1 & 0.01 & 0.06 & 0.04 & 0.11 & 0.04 \\
\hline Sapindaceaea & Matayba sp. & 3 & 0.03 & 0.06 & 0.01 & 0.11 & 0.04 \\
\hline Fabaceae-Faboideae & Hymenolobium sericeum Ducke & 1 & 0.01 & 0.06 & 0.03 & 0.10 & 0.03 \\
\hline $\begin{array}{l}\text { Fabaceae- } \\
\text { Caesalpinioideae }\end{array}$ & Dialium guianense Steud. & 1 & 0.01 & 0.06 & 0.03 & 0.09 & 0.03 \\
\hline Clusiaceae & Vismia sp. & 1 & 0.01 & 0.06 & 0.02 & 0.09 & 0.03 \\
\hline Euphorbiaceae & Alchornea discolor Klotzsch & 2 & 0.02 & 0.06 & 0.01 & 0.09 & 0.03 \\
\hline Urticaceae & Cecropia purpurascens C.C. Berg & 2 & 0.02 & 0.06 & 0.01 & 0.09 & 0.03 \\
\hline Annonaceae & Guatteria poeppigiana & 2 & 0.02 & 0.06 & 0.01 & 0.09 & 0.03 \\
\hline Sapotaceae & Pouteria eugenifolia (Pierre)Baehni & 1 & 0.01 & 0.06 & 0.02 & 0.09 & 0.03 \\
\hline Malpighiaceae & Byrsonima crispa Juss. & 1 & 0.01 & 0.06 & 0.01 & 0.08 & 0.03 \\
\hline $\begin{array}{l}\text { Fabaceae- } \\
\text { Mimosoideae }\end{array}$ & Pithecellobium racemosum Ducke & 1 & 0.01 & 0.06 & 0.01 & 0.08 & 0.03 \\
\hline Malpighiaceae & $\begin{array}{c}\text { Byrsonima duckeana W.R. } \\
\text { Anderson }\end{array}$ & 1 & 0.01 & 0.06 & 0.01 & 0.08 & 0.03 \\
\hline Euphorbiaceae & Croton cajucara Benth. & 1 & 0.01 & 0.06 & 0.01 & 0.08 & 0.03 \\
\hline Celastraceae & Maytenus guyanensis Klotzsch & 1 & 0.01 & 0.06 & 0.01 & 0.08 & 0.03 \\
\hline Melastomataceae & Miconia sp. & 1 & 0.01 & 0.06 & 0.01 & 0.08 & 0.03 \\
\hline Araliaceae & $\begin{array}{c}\text { Schefflera morototoni (Aubl.) } \\
\text { Frondin }\end{array}$ & 1 & 0.01 & 0.06 & 0.01 & 0.08 & 0.03 \\
\hline Malvaceae & Scleronema praecox Ducke & 1 & 0.01 & 0.06 & 0.01 & 0.08 & 0.03 \\
\hline $\begin{array}{l}\text { Fabaceae- } \\
\text { Mimosoideae }\end{array}$ & $\begin{array}{l}\text { Stryphnodendron guianensis } \\
\text { (Aubl.)Benth. }\end{array}$ & 1 & 0.01 & 0.06 & 0.01 & 0.08 & 0.03 \\
\hline $\begin{array}{l}\text { Fabaceae- } \\
\text { Faboideae }\end{array}$ & Swartzia tessmannii Harms & 1 & 0.01 & 0.06 & 0.01 & 0.08 & 0.03 \\
\hline Rubiaceae & Albertia edulis A. Rich. & 1 & 0.01 & 0.06 & 0.00 & 0.07 & 0.02 \\
\hline $\begin{array}{l}\text { Fabaceae- } \\
\text { Faboideae }\end{array}$ & Andira micrantha Ducke & 1 & 0.01 & 0.06 & 0.00 & 0.07 & 0.02 \\
\hline Boraginaceae & Cordia sp. & 1 & 0.01 & 0.06 & 0.00 & 0.07 & 0.02 \\
\hline Chrysobalanaceae & $\begin{array}{c}\text { Couepia canomensis (Mart.) } \\
\text { Benth. ex Hook.f. }\end{array}$ & 1 & 0.01 & 0.06 & 0.00 & 0.07 & 0.02 \\
\hline Lecythidaceae & $\begin{array}{l}\text { Eschweilera rhododendrifolia } \\
\text { (Knuth) A.C.Sm. }\end{array}$ & 1 & 0.01 & 0.06 & 0.00 & 0.07 & 0.02 \\
\hline Myrtaceae & Eugenia diplocampta Diels & 1 & 0.01 & 0.06 & 0.00 & 0.07 & 0.02 \\
\hline Rubiaceae & Ferdinandusa elliptica Pohl. & 1 & 0.01 & 0.06 & 0.00 & 0.07 & 0.02 \\
\hline Meliaceae & Guarea sp. & 1 & 0.01 & 0.06 & 0.00 & 0.07 & 0.02 \\
\hline Quiinaceae & Lacunaria jenmani (Oliv.)Ducke & 1 & 0.01 & 0.06 & 0.00 & 0.07 & 0.02 \\
\hline $\begin{array}{l}\text { Fabaceae- } \\
\text { Caesalpinioideae }\end{array}$ & $\begin{array}{l}\text { Macrolobium angustifolium } \\
\text { (Benth.) R.S.Cowan }\end{array}$ & 1 & 0.01 & 0.06 & 0.00 & 0.07 & 0.02 \\
\hline Simaroubaceae & Simaba cedron Planch. & 1 & 0.01 & 0.06 & 0.00 & 0.07 & 0.02 \\
\hline Monimiaceae & Siparuna amazonica Mart. & 1 & 0.01 & 0.06 & 0.00 & 0.07 & 0.02 \\
\hline Lamiaceae & Vitex cimosa Bert. ex Spreng & 1 & 0.01 & 0.06 & 0.00 & 0.07 & 0.02 \\
\hline Fabaceae-Mimosoideae & Zigia juruana (Harms) L.Rico & 1 & 0.01 & 0.06 & 0.00 & 0.07 & 0.02 \\
\hline Fabaceae- Caesalpinioideae & Zollernia paraensis & 1 & 0.01 & 0.06 & 0.00 & 0.07 & 0.02 \\
\hline
\end{tabular}

\section{DISCUSSION}

In general, the evaluated terra firme forest has floristic composition and structure that are characteristic of this plant type, with most individuals concentrated in few species and few families concentrating the highest percentage of species richness. These results are corroborated by other studies carried out in the same plant typology (PRANCE; RODRIGUES, 1976;
MILLIKEN, 1998; LIMA FILHO et al., 2004; OLIVEIRA; AMARAL, 2004; OLIVEIRA et al., 2008).

The Burseraceae family, despite the less richness in species compared to the Fabaceae, showed higher FIV mainly due to higher number of individuals observed for that family. This family (Burseraceae) appears with the third highest FIV and is the one with the species with the highest IV in the area (Protium hebetatum). 
This shows the great variability in the tropical forests related to the abundance of individuals and diversity in families.

Gama et al. (2005) and Oliveira et al. (2008) reported in their studies that Protium hebetatum and Eschweilera coriacea are the species most commonly recorded in terra firme forests in the Amazônia. Those species have wide geographical distribution and the highest values of abundance. This was also observed in this study, where the density and dominance, in general, contributed to the greater importance of the species in the area.

The greater relative density found for Protium hebetatum has decisively contributed to a higher value of dominance and consequent higher value of IV. The ten species, with the highest IV values showed similar frequency values, with wide distribution in the sampled plots.

The variability in the distribution of the number of species per IV classes, within the plots, especially in the smaller classes can be attributed to the influence of the rare species. Plots 15 and 150 were those that had the highest occurrence of these species in the total set of 15 plots, also being the ones with the highest percentage of exclusive species, occurring with low abundance.

Several studies have reported the occurrence of a significant number of rare species in the Amazonian terra firme forests. These values are similar to those found in this study, on average $34 \%$ of the total number of species (FERRERIA; PRANCE, 1998; OLIVEIRA et al., 2008). Gentry (1988) and Quesada et al. (2009), among others, reported the "preference" for habitats, probably due to variations in soil characteristics, among other factors, as those responsible for the great diversity and dynamics in tropical forests. The high diversity is favored by the occurrence of rare species, whose mechanisms are poorly understood and discussed (Silva, 2010).

In the context of the IV classes, our results show that some species with low abundance have restricted occurrence in the plots and deserve special attention along with rare species ( 1 individual/plot), in order to avoid local extinctions due to management actions. Except for the first lower IV class, it can be considered in general that all plots have species of all IV classes, suggesting that based on this classification, the horizontal structure observed is well represented in the set of plots.

The shape of the distribution curve of the diameters in $\mathrm{J}$-inverted in this study is typical of rain forests with a high proportion of trees with $\mathrm{DBH}<30 \mathrm{~cm}$ (FERREIRA; PRANCE, 1998; LIMA FILHO et al., 2001; HAUGAASEN et al., 2006; GUIMARÃES et al., 2009). The diametric structure found in this work suggests that the area had not suffered major disturbances and the natural dynamics of mortality and recruitment of new individuals, due to the occurrence of small natural clearings may be responsible for the observed distribution (Oliveira et al., 2008). The occurrence of some individuals in classes of diameter greater than $80 \mathrm{~cm}$ also suggests that the forest has not been affected by large disturbances, in which it is observed, for example, individuals of Dinizia excelsa with DBH of approximately $200 \mathrm{~cm}$

\section{CONCLUSIONS}

The use of a large set of data from the 15 permanent plots, allowed us to observe more broadly the variations in the composition and distribution of the species throughout the study area. This was evidenced by the high occurrence of rare species, of species with low density and distribution restricted to some plots. This fact leads us to reflect on how to properly define and discuss the ecological importance of these species, considering that the indexes usually used may not represent their function in the plant community. Studies on the relationship between the species with their occurrence environment can assist in this regard.

Generally, the species and most abundant families, observed in this study are those considered as typical of terra firme dense forest in the Amazon.

\section{REFERENCES}

CIENTEC. Software Mata Nativa 2:

Sistema para Análise Fitossociológica, Elaboração de Inventários e Planos de Manejo de Florestas Nativas. Viçosa, MG: 2006.

CONDÉ, T.M.; TONINI, H. Fitossociologia de uma Floresta Ombrófila Densa na Amazônia Setentrional, Roraima, Brasil. Acta

Amazonica, v.43, n.3, p.247-259, 2013.

Revista Árvore, Viçosa-MG, v.40, n.4, p.603-615, 2016 
CORONADO, E.N.H.; BAKER, T.R.; PHILLIPS, O.L.; PITMAN, N.C.A.; PENNINGTON, R.T.; MARTINEZ, R.V.; MONTEAGUDO, A.; MOGOLLÓN, H.; CARDOZOM, N.D.; RIOS, M.; GARCÍA-VILLACORTA,R.; VALDERRAMA, E.; AHUITE, M.; HUAMANTUPA, I.; NEILL, D.A.; LAURANCE, W.F.; NASCIMENTO, H.E.M.; ALMEIDA, S.S. de; KILLEEN, T.J.; ARROYO, L.; NÚNEZ, P.; ALVARADO, L.F. Multi-scale comparisons of tree composition in Amazonian terra firme forests. Biogeosciences Discussions, v. 6, p.2719-2731, 2009.

FERREIRA, L.V.; PRANCE, G.T. Species richness and floristic composition in four hectares in the Jaú National Park in upland forests in Central Amazonia. Biodiversity and

Conservation, v. 7, n.10, p.1349-1364, 1998.

GAMA, J. R. V.; SOUZA, A.L.; MARTINS, S.V.; SOUZA, D.R. Comparação entre florestas de várzea e de terra firme do Estado do Pará. Revista Árvore, v.29, n.4, p.607-616, 2005.

GENTRY, A.H. Changes in plant community diversity and floristic composition on environmental and geographical gradients. Annals of the Missouri Botanical Garden, 75:1-34, 1988 .

GUIMARÃES, F.J.P. Estrutura de um fragmento florestal no Engenho Humaitá, Catende,

Pernambuco, Brasil. Revista Brasileira de Engenharia Agrícola e Ambiental, v.13, p.940-947, 2009.

HAUGAASEN, T.; PERES, C.A. Floristic, edaphic and structural characteristics of flooded and unflooded forests in the lower Rio Purús region of central Amazonia, Brazil. Acta Amazonica, v.36, n.1, p.25-36, 2006.

INSTITUTO BRASILEIRO DE GEOGRAFIA E ESTATÍSTICA - IBGE. Mapa digital temático de vegetação. Banco de dados Sipam, Brasil. 1999.

LIMA FILHO, D.A., MATOS, F.D.A., AMARAL, I.L., REVILLA, J., COELHO, L.S., RAMOS, J.F., SANTOS, J.L. Inventário florístico de floresta ombrófila densa de terra firme, na região do Rio Urucu-Amazonas, Brasil. Acta Amazonica, n.31, p.565-579, 2001.

Revista Árvore, Viçosa-MG, v.40, n.4, p.603-615, 2016
LIMA FILHO, D. A.; REVILLA, J.; AMARAL, I.L.; MATOS, F.D.de; COELHO, L.S.; RAMOS, J.F.; SILVA, G.B.; GUEDES, J.O. Aspectos florísticos de 13 hectares da área de Cachoeira Porteira-PA. Acta Amazônica, v.34, n.3, p.415-423, 2004.

MILLIKEN, W. Structure and composition of one hectare of central Amazonian terra firme Forest. Biotropica, v.30, n.4, p.530-537, 1998.

MOBOT. Missouri Botanical Garden. St Louis: Estados Unidos. Disponível em: http:// mobot.mobot.orgw3t/search/vas.htm. Acesso em: 12 de fevereiro de 2012 .

MORI, A.S.; BOOM, B. Ecological importance of Myrtaceae in an eastern Brazilian wet forest.

Biotropica, v. 15, n.1, p.68-70, 1983.

MÜller-DOMBOIS, D.; ELLEMBERG, H. Aims and methods for vegetation ecology. New York: John Wiley \& Sons, 1974. 547p.

NOVAES FILHO, J. P.; SELVA, E.C.; COUTO, E.G.; LEHMANN, J.; JOHNSON, M.S.; RIHA, S.J. Distribuição espacial de carbono em solo sob floresta primária na Amazonia Meridional. Revista Árvore, v.31, n.1, p.83-92, 2007.

OLIVEIRA, A.N.; AMARAL, I.L. Florística e fitossociologia de uma floresta de vertente na Amazônia Central, Amazonas, Brasil. Acta Amazônica, v.34, n.1, p.21-34, 2004.

OLIVEIRA, A.N.; AMARAL, I.L.; RAMOS, M.B.P.; NOBRE, A.D.; COUTO, L.B.; SAHDO, R.M. Composição e diversidade florísticoestrutural de um hectare de floresta densa de terra firme na Amazônia Central, Amazonas, Brasil. Acta Amazônica, v. 38, n.4, p.627-642, 2008.

PAS.'Plano Amazônia Sustentável. Governo Federal. 2008. Disponível em: http:// www.mma.gov.br

PHILLIPS O.L.; BAJERM, T.R.M.; ARROYO, L.; HIGUCHI, N.; KILLEN, T.J.; LAURANCE, W.F.; LEWIS, S.L., LLOYD, J.; MALHI, Y.; MONTEAGUDO, A.; NEILL, D.A., VARGAS, P.N.; SILVA, J.N.M.; TERBORG, J., MARTÍNEZ, R.V.; 
ALEIXIADES, M.; ALMEIDA, S.; BROWN, S.; CHAVE, J.; COMISKEY, J.A.; CZIMCZIK, C.I.; FIORE, A.D.; ERWIN, T.; KUEBLER, C.; LAURANCE, S.G.; NASCIMENTO, H.E.M.; OLIVIER, J.; PALACIOS, W.; PATIÑO, S.; PITMAN, N.C.A.; QUESADA, C.A.; SALDIAS, M.; LEZAMA, A.T.; VINCETI, B.Pattern and process in Amazon tree turnover, 1976-2001. HYPERLINK "http:// rstb.royalsocietypublishing.org/" Philosophical Transactions of the Royal Society, v.359, v.1443, p.381-407, 2004.

PITMAN, N.C.M.; TERBORG, S.R.; SILVAN, S.R.; NUNES, P.V.; NEIL, D.A.; CERON, C.E.; PALACIOS, W.A. Dominance and distribution of tree species in upper Amazonia terra firme. Ecology, v. 82, n.11, p. 2101-2117, 2001.

PRANCE, G.T.; RODRIGUES, M.F. Inventário florestal de um hectare de mata de terra firme, km 30 da estrada Manaus-Itacoatiara. Acta Amazônica, v.6, n.1, p.9-35, 1976.

QUESADA, C. A.; LLOYD, J.; SCHWARZ, M.; BAKER, T.R.; PHILLIPS, O.L.; PATIÑO, S.; CZIMCZIK, C.; HODNETT, M.G.; HERRERA, R.; ARNETH, A.; LLOYD, G.; MALHI,Y.; DEZZEO, N.; LUIZÃO, F.J.; SANTOS, A.J.B.; SCHMERLER, J.; ARROYO, L.; SILVEIRA, M.; PRIANTE FILHO, N.; JIMENEZ, E.M.; PAIVA, R.; VÁSQUEZ, R.; NEILL, D.A.; SILVA, N.; PEÑUELA, M.C.; MONTEAGUDO, A.; VÁSQUEZ, R.; PRIETO, A.; RUDAS, A.; ALMEIDA, S.; HIGUCHI, N.; LEZAMA, A.T.; LÓPEZ-GONZÁLEZ, G.; PEACOCK, J.; FYLLAS, N.M.; ALVAREZ DÁVILLA, E.; ERWIN, T.; DI FIORE, A.; CHAO, K.J.; HONORIO, E.;
KILLEEN, T.; PEÑA CRUZ, A.; PITMAN, N.; NUÑEZ VARGAS, P.; SALOMÃ̃, R.; TERBORGH, J.; RAMÍREZ, H. Regional and large-scale patterns in Amazon forest structure and function are mediated by variations in soil physical and chemical properties.

Biogeosciences Discussions, v.6, p.39934057, 2009.

\section{RADAM. Programa de Integração}

Nacional. Levantamentos de Recursos

Naturais. Manaus - DNPM. Brasília: Ministério das Minas e Energia, Brasil, 1978. v.21. 180p.

REGIS, W.D.E. Unidades de Relêvo. In: CALDEIRON, S.S. (Ed.) Recursos naturais e meio ambiente: uma visão do Brasil. Rio de Janeiro: Fundação Instituto Brasileiro de Geografia e Estatística-IBGE, 1993. p.39-45.

RIBEIRO, J.E.L.S.; HOPKINS, M.J.G.; VICENTINI, A.; SOTHERS, C.A.; COSTA, M.A. da S.; BRITO, J.M. de; SOUZA, M.A.D. de; MARTINS, L.H.P.; LOHMANN, L.G.; ASSUNÇÃO, P.A.C.L.; PEREIRA, E.C.; SILVA, C.F.; MESQUITA, M.R.; PROCÓPIO, L.C. Flora da Reserva Ducke. Guia de identificação das plantas vasculares de uma floresta de terra-firme na Amazônia Central. Manaus: Instituto Nacional de Pesquisas da Amazônia, 1999. 793p.

SILVA, K.E. Florística e estrutura espacial: 15 hectares de parcelas permanentes na floresta densa de terra firme na Amazônia Central. 2010. 89f. Tese (Doutorado em Ciência Florestal) - Universidade Federal de Viçosa, MG, 2010.

Revista Árvore, Viçosa-MG, v.40, n.4, p.603-615, 2016 